\title{
Improving the care of people with traumatic brain injury through the Neurotrauma Evidence Translation (NET) program: protocol for a program of research
}

\author{
Sally E Green ${ }^{1 *}$, Marije Bosch², Joanne E McKenzie ${ }^{1}$, Denise A O'Connor ${ }^{1}$, Emma J Tavender ${ }^{2}$, Peter Bragge ${ }^{2}$, \\ Marisa Chau ${ }^{2}$, Veronica Pitt' ${ }^{2}$ Jeffrey $\vee$ Rosenfeld ${ }^{3}$ and Russell L Gruen ${ }^{4}$
}

\begin{abstract}
The Neurotrauma Evidence Translation (NET) program was funded in 2009 to increase the uptake of research evidence in the clinical care of patients who have sustained traumatic brain injury. This paper reports the rationale and plan for this five-year knowledge translation research program. The overarching aims of the program are threefold: to improve outcomes for people with traumatic brain injury; to create a network of neurotrauma clinicians and researchers with expertise in knowledge translation and evidence-based practice; and to contribute knowledge to the field of knowledge translation research. The program comprises a series of interlinked projects spanning varying clinical environments and disciplines relevant to neurotrauma, anchored within four themes representing core knowledge translation activities: reviewing research evidence; understanding practice; developing and testing interventions for practice change; and building capacity for knowledge translation in neurotrauma. The program uses a range of different methods and study designs, including: an evidence fellowship program; conduct of and training in systematic reviews; mixed method study designs to describe and understand factors that influence current practices (e.g., semi-structured interviews and surveys); theory-based methods to develop targeted interventions aiming to change practice; a cluster randomised trial to test the effectiveness of a targeted theoryinformed intervention; stakeholder involvement activities; and knowledge translation events such as consensus conferences.
\end{abstract}

Keywords: Knowledge translation research, Study protocol, Neurotrauma, Traumatic brain injury

\section{Background}

Traumatic Brain Injury (TBI) is an important global health problem. It is defined as injuries caused by external mechanical force to the head, e.g., in motor vehicle accidents, falls, sporting accidents, violent assaults, or blast injuries [1]. Incident estimates range from 108 to 332 hospitalised new cases per 100,000 population per year [2]. There are limited data on the incidence of TBI in low- and middle-income countries; however, epidemiological research from India, with an estimated

\footnotetext{
* Correspondence: sally.green@monash.edu

${ }^{1}$ School of Public Health and Preventive Medicine, Monash University, Melbourne, Australia

Full list of author information is available at the end of the article
}

population size of 1.2 billion, indicates nearly 2 million people sustain TBI each year [3].

TBI can result in long term or lifelong physical, cognitive, behavioural, and emotional consequences. As a result of these consequences, TBI is one of the most disabling injuries [4] and the leading cause of death and disability in children and adults from ages 1 to 44 [5]. The US Centre for Disease Control and Prevention estimates at least 3.17 million Americans, approximately $1.1 \%$ of the US population, are living with long-term disability as a result of TBI [6]. Even mild TBI (mTBI), which accounts for $80 \%$ to $90 \%$ of all TBIs, can cause long-term cognitive problems that may affect a person's ability to perform daily activities and to return to work [7]. Given the incidence and severity of the condition, TBI poses a significant financial burden 
to society [8]. The lifetime cost per case of severe TBI is estimated at \$396,331 USD, with disability and lost productivity costs outweighing medical and rehabilitation costs by a factor of 4 to $1(\$ 330,827$ / $\$ 65,504)$ [9].

Worldwide, much research is conducted relevant to TBI and with the potential to improve outcomes for people with TBI [10]. However, translation of knowledge from research into practice takes considerable time and effort $[11,12]$. Concerted action is needed to facilitate this process, involving individuals, teams, organisations, and systems $[13,14]$. Knowledge translation (KT) is a way to close evidencepractice gaps, and has been defined as 'a dynamic and iterative process that includes the synthesis, dissemination, exchange and ethically sound application of knowledge to improve health, provide more effective health services and products and strengthen the healthcare system' [15].

The care of people with TBI includes many disciplines because patients often have a long journey of care through pre-hospital, hospital, rehabilitation, and community settings [16-18]. At the same time, these are relatively discrete professional communities, which provide opportunities for researchers and 'research users' to collaboratively shape research [19] and so ensure the research conducted is relevant to the TBI community and stakeholders.

In November 2009, the Neurotrauma Evidence Translation (NET) program (www.netprogram.org.au) commenced, funded by the Victorian Government's Transport Accident Commission and Department of Innovation Industry and Regional Development, Australia. This fiveyear program provides opportunity to develop and sustain a coordinated collaborative approach to KT for TBI in Australia. The overall aims of the program are: to improve outcomes for people with TBI; to create a network of neurotrauma clinicians and researchers with expertise in KT and evidence-based practice; and to contribute knowledge to the field of KT research. The program includes a range of integrated activities in the knowledge-to-action-cycle [20]; captured in the following 'themes': reviewing research evidence; understanding practice; developing and testing interventions for practice change, and building capacity for KT in neurotrauma (see Figure 1).

This paper provides the rationale and an overview of the content of the NET program of KT research, including a brief description of the themes and the projects within each theme, their objectives, design, progress to date, and anticipated outcomes. Detailed methods and results of individual projects will be reported separately. At the time of writing of this manuscript, several components had already commenced.

\section{Methods}

\section{Theme one: reviewing research evidence}

For research evidence to be effectively implemented in clinical practice, it first needs to be synthesised and summarised and then made available in formats that are useful and relevant to the local setting [21]. Theme one activities are focused on reviewing the research evidence in TBI, generating knowledge products (e.g., systematic reviews and their derivates, such as consensus statements, and quality indicators), and building capacity to conduct research synthesis. The theme builds on the work of the Global Evidence Mapping Initiative (www. evidencemap.org) [22], which identified priority research questions relevant to TBI via key stakeholder involvement and produced evidence maps to illustrate the breadth and depth of available research addressing these questions. These evidence maps can be used to identify areas in need of synthesis (where evidence exists to guide practice) and 'evidence gaps' where primary research needs to be undertaken.

Theme one of the NET program has two main components: an Evidence Fellowship Program, and the development of locally relevant best practice recommendations.

\section{NET evidence fellowship program}

Knowledge of current research evidence combined with clinical experience and patient preference is pivotal to evidence-based practice. To support evidence-based practice, clinicians need skills to search for, acquire, appraise, and interpret research findings. Barriers to evidence-based practice for clinicians have been welldocumented, and include a lack of time, insufficient resources, and limited skills in accessing and applying research [23]. Fellowships offer clinicians protected time and provide access to resources and technical support that might otherwise be difficult to obtain $[24,25]$.

The objectives of the NET Evidence Fellowship Program are to:

1. build skills and capacity for evidence-based practice within the neurotrauma community through mentoring clinicians to undertake systematic reviews;

2. encourage fellows to become future leaders and knowledge champions;

3. produce systematic reviews in priority neurotrauma topics to inform clinical practice.

To inform the design of the NET Evidence Fellowship Program, a systematic search was conducted in MEDLINE and EMBASE from 1996 to August 2011 to identify literature that described and evaluated evidencebased practice skill-development programs. Relevant papers were reviewed regarding their program concept, results, and experiences. The program was designed to incorporate the main findings from the systematic review and includes: recruitment of clinicians by targeting specific networks or recommendations by referral; access 


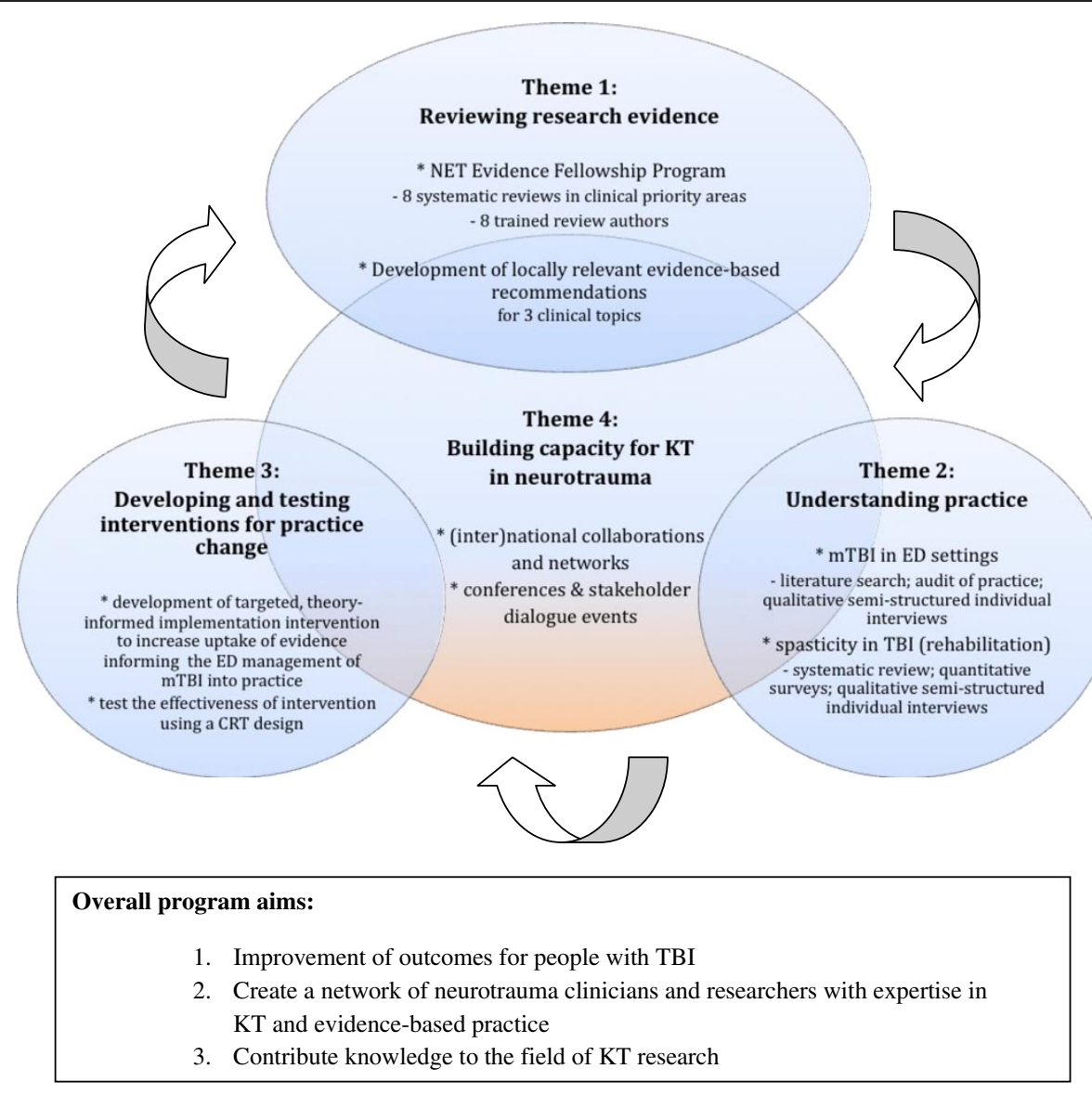

Figure 1 Overview of NET program themes, 'high-level' methods and overall program aims.

to training workshops (e.g., Cochrane Collaboration author training); one-on-one mentoring and training by an experienced systematic reviewer and trainer with skills in searching, critical appraisal, interpretation of results and evidence-based decision making; and the provision of peer and administrative support.

Over the lifetime of the program, eight systematic reviews will be conducted predominantly on the effectiveness of interventions for the management of TBI. Five fellows have been recruited thus far and two protocols have been published [26,27].

\section{Developing locally relevant best practice recommendations}

When evidence has been synthesised, and a reliable body of evidence exists, then efforts need to focus on converting the evidence into formats that are useful to endusers [21]. The second project of theme one focuses on developing best practice recommendations that are actionable, locally applicable, and consistent with best available research-based evidence. In our context, evidence synthesis needs to be suitable to underpin quality indicators and to define and measure best practice. Local adaptation of the evidence is essential [21], as potential dissimilarities in populations, interventions, or outcomes used in research $[28,29]$, or organisational and cultural differences (e.g., beliefs and values) between the research settings of the original studies and the site of implementation [30,31] may influence the relevance and feasibility of implementing a particular 'body of evidence' into a local setting.

Our process therefore encompasses the following steps: identifying current, high quality clinical practice guidelines (CPGs) and extracting recommendations; selecting strong recommendations in key clinical management areas; updating evidence and creating evidence overviews; discussing evidence and producing agreed 'evidence statements'; discussing the relevance of the evidence with local stakeholders; and developing locally applicable actionable best practice recommendations, suitable for use as the basis of quality indicators. The process is reported in full elsewhere [32]. 
This project aims to develop recommendations in three areas of practice, relevant to TBI, in acute and rehabilitation phases of care. To date, the process has been completed for the management of mTBI in Australian emergency department (ED) settings [32,33].

\section{Theme two: understanding practice}

Once evidence-based best practice has been agreed, current practice needs to be determined so as to identify gaps between evidence and practice and establish areas most in need of change [34]. In addition, current KT literature underlines the importance of researching and targeting the factors or determinants that may influence current practice and practice change prior to any efforts to change practice. These determinants may affect individual care providers, teams, organisations, or the wider healthcare system $[35,36]$. Interventions targeting prospectively identified determinants of change are more likely to improve professional practice than no intervention or dissemination of guidelines only [37]. A wide range of methods exist for the identification of these factors $[37,38]$, including the use of theories of behavioural change $[39,40]$. The second theme focuses on understanding current practice and the factors that influence practice change within two clinical areas-management of mTBI in EDs and management of skeletal muscle spasticity following TBI in rehabilitation and community settings.

\section{Management of $\mathrm{mTBI}$ in ED settings}

TBI is a frequent cause of presentation to EDs, and 70\% to $90 \%$ of these are classified as mild $[41,42]$. The National Institutes of Health, USA, has declared that mTBI is a major public health problem and that effort to reduce disability after a mTBI should be a national research priority [43]. As the ED is the main, and often only, point of medical contact for these patients, ED care may have significant impact on the outcomes for these patients. Despite the existence of a variety of evidencebased CPGs on the management of mTBI, studies have shown considerable practice variation in a number of key clinical areas [44-47].

To explore current practice in the ED management of mTBI we triangulated data derived from the following methods: a literature search identifying previous studies on mTBI management in Australian EDs; an audit of management of mTBI in two Victorian EDs; and qualitative interviews with ED staff and directors in a purposeful sample of Victorian EDs to explore reported management. Our descriptions of current practices were compared with agreed local best practice, as determined in theme one [32,33], so as to estimate the presence and extent of any 'knowledge-practice' gaps.
Interviews with ED staff and directors also explored the individual, team, organisational, and system factors that may influence management and implementation of the agreed local best practice recommendations. We interviewed 42 participants (staff and directors) across 13 EDs until data saturation was reached [48]. Sites were selected to reflect both rural and metropolitan EDs. The design of the interview questions and analysis were guided by a theoretical framework [49]. Detailed methods and the findings of the interviews will inform the design of a targeted intervention (theme three) and will be reported in full in subsequent publications.

\section{Managing skeletal muscle spasticity following TBI in rehabilitation and community settings}

Skeletal muscle spasticity is a major physical complication resulting from TBI. There is limited epidemiological data regarding the prevalence of spasticity following TBI, however it has been reported to affect more than one in ten patients with severe TBI [50]. Spasticity and its management have been identified as a priority topic by people with TBI and the multidisciplinary teams managing their care [22]. Interventions for managing skeletal muscle spasticity include pharmacological treatment (e.g., baclofen, botulinum toxin A) and non-pharmacological interventions (e.g., casting, stretching). Currently, little is known about the nature of current management of spasticity following TBI in rehabilitation or community settings.

We plan to explore current practice in the management of spasticity through conduct of a survey of Australian medical and allied health practitioners who manage spasticity following TBI. A random sample of practitioners working in rehabilitation and community care settings will be invited to complete a survey eliciting information about their assessment and treatment of spasticity following TBI. Our estimates of current practice will be compared with systematic review evidence supporting the use of interventions for managing skeletal muscle spasticity following TBI, as determined in theme one [26], so enabling us to estimate the size of the knowledge-practice gap. In-depth interviews with a purposeful sample of medical and allied health practitioners will then be conducted to explore the factors influencing current practice and the determinants of practice change (i.e., the factors that influence the actual change process).

\section{Theme three: developing and testing interventions for practice change}

Once the factors influencing practice and practice change have been identified, the next steps are to develop a KT intervention aiming to address these factors, and to test its effectiveness [51]. We aim to use a theory-informed approach to intervention development [52]. The use of theory 
can offer a generalisable framework for considering effectiveness across different clinical conditions and settings [53]. Given the wide variety of factors that may influence practice change, designing interventions using multiple theoretical perspectives minimises the chance of overlooking important factors [39] and is more likely to promote understanding about how and why change occurs. Research in this theme will build on work we have completed on the development of complex implementation interventions in other settings $[52,54,55]$ and be informed by themes one and two.

Theme three aims to improve outcomes for patients with mTBI presenting to the ED through implementation of the locally relevant best practice recommendations developed in theme one. More specifically, the objectives are:

1. to systematically develop a targeted, theoryinformed, and evidence-based implementation intervention to increase uptake of evidence informing the ED management of mTBI into practice;

2. to test in a cluster randomised trial (CRT) the effectiveness of this intervention in changing practice compared with passive dissemination of the recommendations;

3. to conduct a process evaluation alongside the CRT to understand the pathway of change.

A systematic process to map intervention components to identified determinants of practice change will be used in developing the targeted theory-informed intervention $[51,52,56]$.

We will conduct a CRT to test whether the intervention is effective in improving the uptake of key evidencebased recommendations in the management of mTBI. Hospital EDs will form the clusters. A randomised design is the preferred design to evaluate the effectiveness of an intervention because it minimises bias in estimating intervention effects $[57,58]$. In this study, clusters (hospital EDs) have been chosen because the intervention is targeted at the team of ED staff, and EDs represent patient populations in geographical areas, precluding the use of an individually randomised design $[59,60]$. Intervention sites will receive the targeted, theory-informed intervention, while control sites will receive passive dissemination of the recommendations. A process evaluation will be completed to measure factors along the causal pathway of change and understand why change has or has not taken place [61]. Detailed methods of the CRT will be published as a study protocol.

\section{Theme four: building capacity for KT in neurotrauma}

The overarching and supporting theme of the NET program is to build sustained capacity and infrastructure for $\mathrm{KT}$ in TBI [62], thereby improving patient outcomes as well as contributing to the science of $\mathrm{KT}$ research. To stimulate widespread and sustained uptake of research results into clinical practice beyond those forming the NET program, we aim to contribute to building a culture of research-informed decision making.

Building capacity and developing infrastructure requires investment in resources and structures, such as innovative training, skill development and support for practitioners, and sustained commitment from clinical leaders. Cross sector relationships and partnerships are needed between researchers, clinicians, policy makers, and healthcare consumers to build forums for exchange. To inform this, we need evaluation of what works to implement research into practice; for whom, why, and at what cost. Theme four will harness activities and networks from themes one to three to establish resources and systems, develop a workforce with KT skills, and foster a sustainable neurotrauma KT structure and culture. Specific functions under theme four include the coordination and hosting of three KT conferences over the course of the program, stakeholder dialogue events, and brokerage of relationships across disciplines and sectors through advisory structures and shared prioritisation.

The NET program has a program-wide multidisciplinary steering committee, which provides strategic direction to the program, offers practical support towards achieving the overarching aims of the program, and fosters links between the NET program and its identified stakeholder groups-the neurotrauma clinical community, consumers, policy makers, and funders. A number of networks and collaborations have been formed spanning Australia, Canada, USA, and UK, and these teams are exploring new neurotrauma $\mathrm{KT}$ initiatives that build upon the activities in the NET program.

\section{Conclusion}

The NET program is a coordinated approach to KT in TBI in Australia. The program comprises a series of interlinked projects spanning varying clinical environments and disciplines, anchored within four themes representing core KT activities: reviewing research evidence; understanding practice; developing and testing interventions for practice change, and building capacity for $\mathrm{KT}$ in neurotrauma. The program aims to contribute to improving care for patients with TBI in Australia, and to contribute to the science of $\mathrm{KT}$ research.

\section{Abbreviations}

CPG: Clinical Practice Guideline; CRT: Cluster Randomised Trial; ED: Emergency Department; (m)TBI: (mild) Traumatic Brain Injury; NET: Neurotrauma Evidence Translation; KT: Knowledge Translation.

\section{Competing interests}

Denise O'Connor is an Associate Editor for Implementation Science. The authors declare they have no other competing interests. 


\section{Authors' contributions}

Sally Green and Russell Gruen conceived of the program and wrote the original grant proposal. Joanne McKenzie and Denise O'Connor contributed to sections of the original grant proposal. Marije Bosch wrote the first draft of the manuscript and prepared the revised versions. All other authors critically reviewed and contributed to draft revisions, and read and approved the final version of the manuscript.

\section{Acknowledgements}

This protocol is based on the grant titled 'Improving evidence-based care and the outcomes of patients with traumatic brain injury and spinal cord injury through a program to facilitate knowledge transfer and exchange.' This study was funded by the Victorian Transport Accident Commission, Australia. We thank Ms Kate Phillips and the wider investigator team: Dr Mark Bayley; Dr Heather Buchan; Professor Peter Cameron; Professor Jamie Cooper; Dr David Cooksley; Professor Niki Ellis; Associate Professor Mark Fitzgerald; Professor Jill Francis; Professor Jeremy Grimshaw; Dr Sophie Hill; Ms Sue Huckson; Dr Tony Joseph; Dr Fary Khan; Dr Jonathan Knott; Professor John Lavis; Associate Professor Shawn Marshall; Professor Susan Michie; Associate Professor Peter Morley; Dr Andrew Pearce; Professor Jennie Ponsford; Mr Nick Rushworth; Dr Elisabeth (Lisa) Sherry.

\section{Author details}

${ }^{1}$ School of Public Health and Preventive Medicine, Monash University, Melbourne, Australia. ${ }^{2}$ Department of Surgery, Monash University / National Trauma Research Institute, Melbourne, Australia. ${ }^{3}$ Department of Surgery, Monash University / Department of Neurosurgery, The Alfred Hospital / National Trauma Research Institute, Melbourne, Australia. ${ }^{4}$ Department of Trauma, The Alfred Hospital / Department of Surgery, Monash University / National Trauma Research Institute, Melbourne, Australia.

Received: 29 May 2012 Accepted: 27 July 2012

Published: 7 August 2012

\section{References}

1. Maas Al, Stocchetti N, Bullock R: Moderate and severe traumatic brain injury in adults. Lancet Neurol 2008, 7(8):728-741.

2. Abelson-Mitchell $\mathrm{N}$ : Epidemiology and prevention of head injuries: literature review. J Clin Nurs 2008, 17(1):46-57.

3. Gururaj G, Kolluri S, Chandramouli B, Subbaskrishna D, Kraus J: Traumatic Brain Injury. In National Institute of Mental Health \& Neuro Sciences, Publication no. 61. India: Banglore, 560029; 2005.

4. Leibson CL, Brown AW, Ransom JE, Diehl NN, Perkins PK, Mandrekar J, Malec JF: Incidence of traumatic brain injury across the full disease spectrum: a population-based medical record review study. Epidemiology 2011, 22(6):836-844

5. TB/ statistics. https://www.braintrauma.org/tbi-fags/tbi-statistics/.

6. Summers CR, Ivins B, Schwab KA: Traumatic brain injury in the United States: an epidemiologic overview. Mt Sinai J Med 2009, 76(2):105-110.

7. Faux S, Sheedy J: A prospective controlled study in the prevalence of posttraumatic headache following mild traumatic brain injury. Pain Med 2008, 9(8):1001-1011.

8. Polinder S, Meerding WJ, Mulder S, Petridou E, van Beeck E: Assessing the burden of injury in six European countries. Bull World Health Organ 2007 85(1):27-34

9. Faul M, Wald MM, Rutland-Brown W, Sullivent EE, Sattin RW: Using a costbenefit analysis to estimate outcomes of a clinical treatment guideline: testing the Brain Trauma Foundation guidelines for the treatment of severe traumatic brain injury. J Trauma 2007, 63(6):1271-1278.

10. Bragge P, Chau M, Pitt VJ, Bayley M, Eng JJ, Teasell R, Wolfe DL, Gruen RL: An overview of published research about the acute care and rehabilitation of traumatic brain injured and spinal cord injured patients. J Neurotrauma 2012. doi:10.1089/neu.2011.2193 [Epub ahead of print].

11. Morris ZS, Wooding S, Grant J: The answer is 17 years, what is the question: understanding time lags in translational research. $J R$ Soc Med 2011, 104(12):510-520.

12. Woolf $\mathrm{SH}$ : The meaning of translational research and why it matters. JAMA 2008, 299(2):211-213.

13. Grol $R$, Wensing $M$ : What drives change? Barriers to and incentives for achieving evidence-based practice. Med J Aust 2004, 180(6 Suppl):S57-S60
14. Shortell SM, Singer SJ: Improving patient safety by taking systems seriously. JAMA 2008, 299(4):445-447.

15. Straus SE, Tetroe J, Graham ID: Knowledge to action: what it is and what it isn't. In Knowledge translation in health care: moving from evidence to practice. Edited by Straus SE, Tetroe J, Graham ID. Chichester: Blackwell Publishing Ltd; 2009.

16. Khan F, Baguley IJ, Cameron ID: 4: Rehabilitation after traumatic brain injury. Med J Aust 2003, 178(6):290-295.

17. Trevena $L$, Cameron I: Traumatic brain injury - long term care of patients in general practice. Aust Fam Physician 2011, 40(12):956-961.

18. O'Callaghan AM, McAllister L, Wilson L: Sixteen years on: has quality of care for rural and non-compensable traumatic brain injury clients improved? Aust J Rural Health 2009, 17(3):119-123.

19. Graham ID, Tetroe J: How to translate health research knowledge into effective healthcare action. Healthc Q 2007, 10(3):20-22.

20. Graham ID, Logan J, Harrison MB, Straus SE, Tetroe J, Caswell W, Robinson $\mathrm{N}$ : Lost in knowledge translation: time for a map? J Contin Educ Health Prof 2006, 26(1):13-24.

21. Straus SE, Tetroe J, Graham I: Defining knowledge translation. CMAJ 2009, 181(3-4):165-168.

22. Bragge P, Clavisi O, Turner T, Tavender E, Collie A, Gruen RL: The global evidence mapping initiative: scoping research in broad topic areas. BMC Med Res Methodol 2011, 11:92.

23. Sitzia J: Barriers to research utilisation: the clinical setting and nurses themselves. Intensive Crit Care Nurs 2002, 18(4):230-243.

24. Short J, McDonald S, Turner T, Martis R: Improving capacity for evidencebased practice in South East Asia: evaluating the role of research fellowships in the SEA-ORCHID Project. BMC Med Educ 2010, 10:37.

25. Chau M, Pitt V, Bragge P, Green S, Gruen R: Mentoring clinicians in conducting systematic reviews through a clinical fellowship program. Abstracts of the 19th Cochrane Colloquium. October 19-22, 2011 Madrid, Spain. Cochrane Database Syst Rev 2011, Suppl 3:CD000003.

26. Phillips K, Pitt V, O'Connor D, Gruen R: Interventions for managing skeletal muscle spasticity following traumatic brain injury (Protocol). Cochrane Database Syst Rev 2011, (Issue 1. Art. No). doi:10.1002/14651858. CD008929.

27. Gauden AJ, Pitt V, Gruen RL: Excitatory amino acid inhibitors for traumatic brain injury (Protocol). Cochrane Database Syst Rev 2012, (Issue 2. Art. No). doi:10.1002/14651858.CD009661.

28. McAlister FA, van Diepen S, Padwal RS, Johnson JA, Majumdar SR: How evidence-based are the recommendations in evidence-based guidelines? PLoS Med 2007, 4(8):e250.

29. O'Connor CM, Fiuzat M, Swedberg K, Caron M, Koch B, Carson PE, Gattis-Stough W, Davis GW, Bristow MR: Influence of global region on outcomes in heart failure beta-blocker trials. J Am Coll Cardiol 2011, 58(9):915-922.

30. Marshall MN, Shekelle PG, McGlynn EA, Campbell S, Brook RH, Roland $\mathrm{MO}$ : Can health care quality indicators be transferred between countries? Qual Saf Health Care 2003, 12(1):8-12.

31. Raine R, Sanderson C, Hutchings A, Carter S, Larkin K, Black N: An experimental study of determinants of group judgments in clinical guideline development. Lancet 2004, 364(9432):429-437.

32. Bosch M, Tavender E, Bragge P, Gruen R, Green S: How to define 'best practice' for use in knowledge translation research: a practical, stepped and interactive process. J Eval Clin Pract 2012, doi:10.1111/j.1365-2753.2012.01835.x [Epub ahead of print].

33. Tavender EJ, Bosch M, Green S, O'Connor D, Pitt V, Phillips K, Bragge P, Gruen RL: Quality and consistency of guidelines for the management of mild traumatic brain injury in the emergency department. Acad Emerg Med 2011, 18(8):880-889.

34. Kitson A, Straus SE: The knowledge-to-action cycle: identifying the gaps. CMAJ 2010, 182(2):E73-77.

35. Grol RP, Bosch MC, Hulscher ME, Eccles MP, Wensing M: Planning and studying improvement in patient care: the use of theoretical perspectives. Milbank Q 2007, 85(1):93-138.

36. Ferlie EB, Shortell SM: Improving the quality of health care in the United Kingdom and the United States: a framework for change. Milbank Q 2001, 79(2):281-315.

37. Baker R, Camosso-Stefinovic J, Gillies C, Shaw EJ, Cheater F, Flottorp S, Robertson N: Tailored interventions to overcome identified barriers to change: effects on professional practice and health care outcomes. 
Cochrane Database Syst Rev 2010, (Issue 3. Art. No). 10.1002/14651858. CD005470.pub2.CD005470.

38. Bosch $M$, van der Weijden $T$, Wensing $M$, Grol R: Tailoring quality improvement interventions to identified barriers: a multiple case analysis. J Eval Clin Pract 2007, 13(2):161-168.

39. The Improved Clinical Effectiveness through Behavioural Research Group (ICEBeRG): Designing theoretically-informed implementation interventions. Implement Sci 2006, 1:4.

40. Foy R, Ovretveit J, Shekelle PG, Pronovost PJ, Taylor SL, Dy S, Hempel S, McDonald KM, Rubenstein LV, Wachter RM: The role of theory in research to develop and evaluate the implementation of patient safety practices. BMJ Qual Saf 2011, 20(5):453-459.

41. Bruns JJ Jr, Jagoda AS: Mild traumatic brain injury. Mt Sinai J Med 2009, 76(2):129-137.

42. Cassidy JD, Carroll L, Peloso PM, Borg J, von Holst H, Holm L, Kraus J, Coronado VG: Incidence, risk factors and prevention of mild traumatic brain injury: results of the WHO collaborating centre task force on mild traumatic brain injury. J Rehabil Med 2004, (43 Suppl):28-60.

43. Consensus conference. Rehabilitation of persons with traumatic brain injury. NIH consensus development panel on rehabilitation of persons with traumatic brain injury. JAMA 1999, 282(10):974-983.

44. Bellner J, Ingebrigtsen T, Romner B: Survey of the management of patients with minor head injuries in hospitals in Sweden. Acta Neurol Scand 1999, 100(6):355-359.

45. Miller L, Kent RM, Tennant A: Audit of head injury management in accident and emergency at two hospitals: implications for NICE CT guidelines. BMC Health Serv Res 2004, 4(1):7.

46. Shravat BP, Huseyin TS, Hynes KA: NICE guideline for the management of head injury: an audit demonstrating its impact on a district general hospital, with a cost analysis for England and Wales. Emerg Med J 2006, 23(2):109-113.

47. Stiell IG, Wells GA, Vandemheen K, Laupacis A, Brison R, Eisenhauer MA, Greenberg GH, MacPhail I, McKnight RD, Reardon M, et al: Variation in ED use of computed tomography for patients with minor head injury. Ann Emerg Med 1997, 30(1):14-22.

48. Glaser BG, Strauss A: The discovery of grounded theory: strategies for qualitative research. Chicago, IL: Aldine; 1967.

49. Michie S, Johnston M, Abraham C, Lawton R, Parker D, Walker A: Making psychological theory useful for implementing evidence based practice: a consensus approach. Qual Saf Health Care 2005, 14(1):26-33.

50. Singer BJ, Jegasothy GM, Singer KP, Allison GT, Dunne JW: Incidence of ankle contracture after moderate to severe acquired brain injury. Arch Phys Med Rehabil 2004, 85(9):1465-1469.

51. Wensing M, Bosch M, Grol R: Developing and selecting interventions for translating knowledge to action. CMAJ 2010, 182(2):E85-88.

52. French SD, Green SE, O'Connor DA, McKenzie JE, Francis JJ, Michie S, Buchbinder R, Schattner P, Spike N, Grimshaw JM: Developing theoryinformed behaviour change interventions to implement evidence into practice: a systematic approach using the theoretical domains framework. Implement Sci 2012, 7(1):38.

53. Eccles M, Grimshaw J, Walker A, Johnston M, Pitts N: Changing the behavior of healthcare professionals: the use of theory in promoting the uptake of research findings. J Clin Epidemiol 2005, 58(2):107-112.

54. McKenzie JE, French SD, O'Connor DA, Grimshaw JM, Mortimer D, Michie S, Francis J, Spike N, Schattner P, Kent PM, et al: IMPLEmenting a clinical practice guideline for acute low back pain evidence-based manageMENT in general practice (IMPLEMENT): cluster randomised controlled trial study protocol. Implement Sci 2008, 3:11.

55. McKenzie JE, O'Connor DA, Page MJ, Mortimer DS, French SD, Walker BF, Keating JL, Grimshaw JM, Michie S, Francis JJ, et al: Improving the care for people with acute low-back pain by allied health professionals (the ALIGN trial): a cluster randomised trial protocol. Implement Sci 2010, 5:86.

56. Michie S, Johnston M, Francis J, Hardeman W, Eccles M: From theory to intervention: mapping theoretically derived behavioural determinants to behaviour change techniques. Appl Psychol: Int Rev 2008, 57(4):660-680.

57. Grimes DA, Schulz KF: An overview of clinical research: the lay of the land. Lancet 2002, 359(9300):57-61.

58. Glynn RJ, Brookhart MA, Stedman M, Avorn J, Solomon DH: Design of cluster-randomized trials of quality improvement interventions aimed at medical care providers. Med Care 2007, 45(10 Suppl 2):S38-43.
59. Edwards SJ, Braunholtz DA, Lilford RJ, Stevens AJ: Ethical issues in the design and conduct of cluster randomised controlled trials. BMJ 1999, 318(7195):1407-1409.

60. Walker AE, Campbell MK, Grimshaw JM: A recruitment strategy for cluster randomized trials in secondary care settings. J Eval Clin Pract 2000, 6 (2):185-192.

61. Grimshaw JM, Zwarenstein M, Tetroe JM, Godin G, Graham ID, Lemyre L, Eccles MP, Johnston M, Francis JJ, Hux J, et al: Looking inside the black box: a theory-based process evaluation alongside a randomised controlled trial of printed educational materials (the Ontario printed educational message, OPEM) to improve referral and prescribing practices in primary care in Ontario, Canada. Implement Sci 2007, 2:38,

62. Potter $C$, Brough R: Systemic capacity building: a hierarchy of needs. Health Policy Plan 2004, 19(5):336-345.

doi:10.1186/1748-5908-7-74

Cite this article as: Green et al: Improving the care of people with traumatic brain injury through the Neurotrauma Evidence Translation (NET) program: protocol for a program of research. Implementation Science 2012 7:74

\section{Submit your next manuscript to BioMed Central and take full advantage of:}

- Convenient online submission

- Thorough peer review

- No space constraints or color figure charges

- Immediate publication on acceptance

- Inclusion in PubMed, CAS, Scopus and Google Scholar

- Research which is freely available for redistribution 